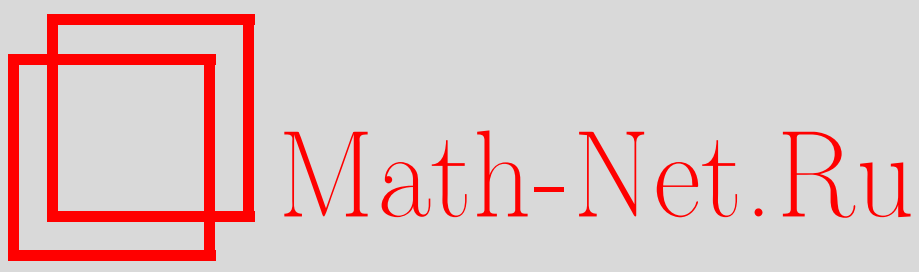

С. С. Платонов, Приближения на компактных симметрических пространствах ранга 1, Матем. сб., 1997, том 188, номер 5, 113-130

DOI: https://doi.org/10.4213/sm233

Использование Общероссийского математического портала Math-Net.Ru подразумевает, что вы прочитали и согласны с пользовательским соглашением http://www . mathnet.ru/rus/agreement

Параметры загрузки:

IP : 52.90 .164 .192

26 апреля 2023 г., 14:03:39 
УДК 517.518

\author{
С. С. Платонов
}

\title{
Приближения на компактных симметрических пространствах ранга 1
}

\begin{abstract}
На произвольном компактном римановом симметрическом пространстве $M$ ранга 1 вводятся классы Никольского $H_{p}^{r}(M)$, определяемые с помощью разностей вдоль геодезических, и дается описание этих пространств в терминах наилучших приближений полиномами по сферическим гармоникам на $M$, т.е. линейными комбинациями собственных функций оператора Лапласа-Бельтрами на $M$. Результаты статьи обобщают результаты С. М. Никольского и П. И. Лизоркина о приближении функций на сфере $S^{n}$.

Библиограф̆ия: 16 названий.
\end{abstract}

\section{§1. Введение и формулировка результатов}

В последние годы выходит много работ, посвященных изучению различных задач теории приближений на $n$-мерной сфере $S^{n}$ (см. [1]-[5] и цитированную там литературу). Естественным более широким классом пространств, на которых можно ставить аналогичные задачи, является множество всех компактных симметрических пространств ранга 1 (КРОСП-ов, по терминологии книги [8]). Для этих пространств уже получены некоторые результаты (см. [6], [7]), но остается еще много задач. Настоящая работа посвяшена перенесению результатов С.М. Никольского и П.И. Лизоркина [1]-[3] на произвольный КРОСП.

Пусть $M$ - КРОСП. Хорошо известна полная классификация всех КРОСП-ов. Их всего четыре серии (индекс $n$ всюду означает размерность пространства): сферы $S^{n}(n=1,2, \ldots)$, вешественное, комплексное и кватернионное проективные пространства $\left(P^{n}(\mathbb{R})(n=2,3, \ldots), P^{n}(\mathbb{C})(n=4,6, \ldots), P^{n}(\mathbb{H})\right.$ $(n=8,12,16, \ldots))$ и одно особое пространство - эллиптическая плоскость Кэли $P^{16}($ Cay $)$. Так как задачи гармонического анализа на $P^{n}(\mathbb{R})$ легко сводятся к соответствуюшим задачам на $S^{n}$, то будем считать, что $M \neq P^{n}(\mathbb{R})$.

$M$ является римановым многообразием, пусть $d x$ - элемент объема на $M$. Как обычно, через $L_{p}(M)$ обозначим банахово пространство, состоящее из измеримых комплекснозначных функций $f(x)$ на $M$ с конечной нормой

$$
\|f\|_{p}=\left(\int_{M}|f(x)|^{p} d x\right)^{1 / p}, \quad 1 \leqslant p<\infty .
$$

Пусть $L_{\infty}(M)=C(M)$ - пространство непрерывных на $M$ функций $f$ с нормой

$$
\|f\|_{\infty}=\|f\|_{C}=\max _{x \in M}|f(x)| .
$$


Пусть $O$ - фиксированная точка пространства $M$. Пространство $M$ можно реализовать как факторпространство $U / K$, где $U$ - связная компактная группа Ли (связная компонента единицы в группе изометрий пространства $M), K$ - стационарная подгруппа точки $O$. При этом функции $f(x)$ на $M$ отождествляются с функциями $f(u)$ на $U$, удовлетворяющими дополнительному условию

$$
f(u k)=f(u) \quad \forall k \in K .
$$

Пусть $T: U \rightarrow G L(E)$ - неприводимое представление группы $U$ в конечномерном векторном пространстве $E,\langle\cdot, \cdot\rangle$ - инвариантная эрмитова форма в пространстве $E$. Представление называется сферическим $[9$, гл. $4, \S 1]$, если в пространстве $E$ существует ненулевой $K$-инвариантный вектор $e_{0}$ (т.е. $\left.T(k) e_{0}=e_{0} \forall k \in K\right)$. Такой вектор единственный с точностью до умножения на число. Будем считать, что $\left|e_{0}\right|=\left\langle e_{0}, e_{0}\right\rangle^{1 / 2}=1$. Функция $\varphi(u)=\left\langle T(u) e_{0}, e_{0}\right\rangle$ называется сферической функиией, соответствуюшей представлению $T$.

Приведем некоторые сведения из теории симметрических пространств по книге [9]. Обозначим через $\mathfrak{u}$ и $\mathfrak{k}$ алгебры Ли групп $U$ и $K$, соответственно. Пусть $\mathfrak{u}=\mathfrak{p} \oplus \mathfrak{k}$ - разложение Картана алгебры $\mathfrak{u}$ (т.е. $\mathfrak{p}$ - ортогональное дополнение к $\mathfrak{k}$ относительно формы Киллинга на алгебре Ли $\mathfrak{u})$. Выберем в $\mathfrak{p}$ максимальную абелеву подалгебру $\mathfrak{a}$ и расширим $\mathfrak{a}$ до максимальной абелевой подалгебры $\mathfrak{h}$ в $\mathfrak{u}$. Тогда $\mathfrak{h}=\mathfrak{a}+\mathfrak{h} \cap \mathfrak{k}$ (см. [9, с. 593]). Если $M$ - симметрическое пространство ранга 1 , то $\operatorname{dim} \mathfrak{a}=1$.

Для $H \in \mathfrak{a}$ обозначим через $\tau_{H}$ линейное преобразование $Y \rightarrow[H,[H, Y]]$ пространства p. Собственные значения оператора $\tau_{H}$ таковы: $0, \alpha(H)^{2}$ и, возможно, $(\alpha(H) / 2)^{2}$, где $\alpha(H)$ - некоторая линейная функция на $\mathfrak{a}$. Все пространство $\mathfrak{p}$ раскладывается в прямую сумму собственных подпространств оператора $\tau_{H}$, соответствуюших собственньм значениям $0, \alpha(H)^{2}$ и $(\alpha(H) / 2)^{2}$ :

$$
\mathfrak{p}=\mathfrak{a}+\mathfrak{p}_{\alpha}+\mathfrak{p}_{\alpha / 2}
$$

Пусть $a=\operatorname{dim} \mathfrak{p}_{\alpha / 2}, b=\operatorname{dim} \mathfrak{p}_{\alpha}$. Числа $a$ и $b$ часто встречаются в различных формулах, связанных с симметрическими пространствами. Приведем их значения для конкретных пространств $[9$, гл. $1, \S 4$, п. 2].

$$
\begin{array}{ll}
M=\mathbb{S}^{n}: & a=0, \quad b=n-1 ; \\
M=P^{n}(\mathbb{C}): & a=n-2, \quad b=1 ; \\
M=P^{n}(\mathbb{H}): & a=n-4, \quad b=3 ; \\
M=P^{16}(\text { Cay }): & a=8, \quad b=7 .
\end{array}
$$

Каждое неприводимое представление групшы $U$ однозначно задается своим старшим весом $\lambda$, который является линейным функционалом на $\mathfrak{h}$. Пусть $\Lambda_{0}-$ множество всех старших весов сферических представлений группы $U$. Если $M=U / K-$ КРОCП, то известно $[9$, гл. V, $\S 4$, п. 1$]$, что сушествует старший вес $\mu \in \Lambda_{0}$ такой, что

$$
\Lambda_{0}=\{\lambda=l \mu, l=0,1,2, \ldots\}
$$


(т.е. $\Lambda_{0}$ состоит из старших весов, кратных $\mu$ ). Обозначим через $T^{l}$ сферическое представление со старшим весом $l \mu\left(l \in \mathbb{Z}_{+}, \mathbb{Z}_{+}-\right.$множество неотрицательных целых чисел), $E^{l}$ - пространство этого представления, $d_{l}=\operatorname{dim} E^{l},\langle\cdot, \cdot\rangle-$ инвариантная эрмитова форма в $E^{l}$. Выберем в $E^{l}$ ортонормированный базис $e_{0}^{l}, e_{1}^{l}, \ldots$, причем пусть $e_{0}-K$-инвариантный вектор в $E^{l}$. Функции

$$
\tau_{i j}^{l}(u)=\left\langle T^{l}(u) e_{i}^{l}, e_{j}^{l}\right\rangle, \quad i, j=0,1, \ldots, d_{l}-1,
$$

называются матричными әлементами представления $T^{l}$, в частности, $\varphi^{l}(u)=$ $\tau_{00}^{l}(u)$ - сферическая функция представления $T^{l}$. Функции $\tau_{i 0}^{l}(u)\left(l \in \mathbb{Z}_{+}\right.$, $i=0,1, \ldots l-1)$ образуют полную ортогональную (но не ортонормированную) систему в гильбертовом пространстве $L_{2}(M)$ и обычно используются для изучения различных вопросов гармонического анализа на $M$. Отметим еще, что линейная оболочка функций $\tau_{i 0}^{l}(u)$ при фиксированном $l$ и $0 \leqslant i \leqslant d_{l}-1$ совпадает с собственным подпространством для оператора Лапласа-Бельтрами на многообразии $M$, отвечающим некоторому собственному значению $\lambda_{l}$. При этом $\lambda_{l} \leqslant 0,\left|\lambda_{l+1}\right|>\left|\lambda_{l}\right|$ и числа $\lambda_{l}$ образуют спектр оператора Лапласа-Бельтрами. Можно выписать и явные формулы для чисел $\lambda_{l}[8$, гл. 8], если риманова метрика на $M$ нормирована так, что длина максимальной геодезической равна $2 \pi$ (см. ниже):

$$
\begin{array}{ll}
\text { для } S^{n}: & \lambda_{l}=l(l+n-1) ; \\
\text { для } P^{n}(\mathbb{C}): & \lambda_{l}=l(l+n) ; \\
\text { для } P^{n}(\mathbb{H}): & \lambda_{l}=l(l+2 n+1) ; \\
\text { для } P^{16}(\text { Cay }): & \lambda_{l}=l(l+11) .
\end{array}
$$

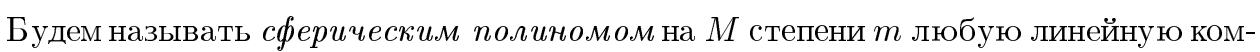
бинацию функций $\tau_{i 0}^{l}$ при $l \leqslant m$. Обозначим через $\mathscr{P}_{m}(M)$ множество сферических полиномов степени $m$. В частности, при $M=S^{n}$ получаем обычные сферические полиномы. Естественно возникает задача о приближении функций на КРОСП-е $M$ сферическими функциями. Здесь удается получить результаты, аналогичные результатам С.М. Никольского и П.И. Лизоркина [1]-[3] о приближениях на $S^{n}$.

Для $x, y \in M$ пусть $|x y|$ - расстояние между точками $x$ и $y$. Через $L$ обозначим наибольшее расстояние между точками пространства $M$ (т.е. $L$ - диаметр пространства $M)$. Известно (см. [10, гл. IX, $\S 5])$, что если $|x y|<L$, то через точки $x$ и $y$ можно провести единственную геодезическую. Кроме того, все геодезические на $M$ замкнуты и имеют одинаковую длину $2 L$. Риманова метрика на $M$ определена с точностью до умножения на положительное число. Для удобства нормируем риманову метрику так, чтобы $L=\pi$.

Для $x \in M$ через $T_{x} M$ будем обозначать множество касательных векторов к многообразию $M$ в точке $x$. Пусть $x \in M$ и $\xi$ - единичньй касательньй вектор в точке $x$. Обозначим через $\gamma(x, \xi ; s)$ натурально параметризованную геодезическую, выходящую из точки $x$ в направлении вектора $\xi$, т.е. $\gamma(x, \xi ; 0)=x$ и $\left.\frac{d}{d s} \gamma(x, \xi ; s)\right|_{s=0}=\xi$, где $\frac{d}{d s} \gamma(s)$ - касательный вектор к кривой $\gamma(s)$. 
$k$-я разность функции $f(x)$ в точке $x$ в направлении $\xi$ с шагом $t$ определяется равенством

$$
{ }_{\xi} \Delta_{t}^{k} f(x)=\sum_{j=0}^{k}(-1)^{k-j} C_{k}^{j} f(\gamma(x, \xi ; j t)) .
$$

Пусть

$$
S(x)=\left\{\xi \in T_{x} M:|\xi|=1\right\}
$$

- единичная сфера в касательном пространстве $T_{x} M$. Будем обозначать через $d \mu(\xi)$ элемент объема сферы $S(x)$, а через $\sigma$ - полньй объем сферы $S(x)$.

Определим новую разность

$$
{ }^{*} \Delta_{t}^{k} f(x)=\frac{1}{\sigma} \int_{S(x)} \xi \Delta_{t}^{k} f(x) d \mu(\xi) .
$$

По определению функция $f$ принадлежит пространству $H_{p}^{r}(M)$, если $f \in L_{p}(M)$ и

$$
\left\|^{*} \Delta_{t}^{k} f(x O)\right\|_{p} \leqslant c t^{r} \quad \forall t>0,
$$

где $c$ не зависит от $t$ и $k>r>0$. Норма в пространстве $H_{p}^{r}(M)$ определяется так:

$$
\|f\|_{H_{p}^{r}(M)}=\|f\|_{p}+c_{f},
$$

где $c_{f}$ - наименьшая константа в неравенстве (1.5).

Наилучшим приближением функции $f$ в метрике $L_{p}(1 \leqslant p \leqslant \infty)$ при помоши сферических полиномов степени $m$ назьвается число

$$
E_{m}(f)_{p}=\min _{\Phi \in \mathscr{P}_{m}}\|f-\Phi\|_{p}
$$

Teоpema 1. a) Ecлu $f \in H_{p}^{r}(M)$, mo

$$
E_{m}(f)_{p} \leqslant \frac{c\|f\|_{H_{p}^{r}(M)}}{m^{r}}, \quad m=1,2,3, \ldots
$$

где постоянная с не зависит от $f$ и $\mathrm{m}$.

б) Обратно, если

$$
E_{m}(f)_{p} \leqslant \frac{c_{1}}{m^{r}}, \quad m=1,2
$$

mo $f \in H_{p}^{r}(M) u$

$$
\|f\|_{H_{p}^{r}(M)} \leqslant c_{2}\left(\|f\|_{p}+c_{1}\right)
$$

где постоянная $c_{2}$ не зависит от $f$.

Для сферы $S^{n}$ теорема 1 доказана в [3], поэтому будем считать, что $M-$ одно из пространств $P^{n}(\mathbb{C}), P^{n}(\mathbb{H}), P^{16}($ Cay $)$.

Доказательство теоремы 1 является основной целью настоящей работы. В $\S 2$ для функции $f \in H_{p}^{r}(M)$ строится последовательность приближающих ее сферических полиномов и доказывается прямая часть теоремы $1 . \mathrm{B} \S 3$ доказываются необходимые неравенства типа Бернштейна (см. леммы 3.4 и 3.5 ) и обратная часть теоремы 1. 


\section{§2. Построение сферических полиномов}

Пусть $S_{M}(x ; t)=\left\{x^{\prime} \in M:\left|x x^{\prime}\right|=t\right\}$ - сфера радиуса $t$ с центром в точке $x$ в пространстве $M$. Пусть $d \sigma\left(x^{\prime}\right)$ - элемент объема сферы $S_{M}(x ; t), \sigma(t)$ - полный объем всей сферы $S_{M}(x ; t)$. Аналогично [3] определим оператор сдвига $S_{t} f(x)$ функции $f \in L_{p}(M)$ с шагом $t$

$$
S_{t} f(x)=\frac{1}{\sigma(t)} \int_{S_{M}(x ; t)} f\left(x^{\prime}\right) d \sigma\left(x^{\prime}\right), \quad 0<t<\pi .
$$

При этом, как и для случая $M=S^{n}$, справедливо неравенство

$$
\left\|S_{t} f\right\|_{p} \leqslant\|f\|_{p}
$$

Если воспользоваться реализацией $M=U / K$, то касательное пространство к $M$ в точке $O$ естественньм образом отождествляется с векторным пространством $\mathfrak{p}(\mathfrak{u}=\mathfrak{k}+\mathfrak{p}-$ разложение Картана, все обозначения взяты из $\S 1)$. В частности, для $X \in \mathfrak{p}$ пусть $|X|$ - длина этого вектора как касательного вектора к $M$. В одномерном пространстве $\mathfrak{a}$ выберем базисный вектор $H$ такой, что $|H|=1$. Отображение $t \mapsto R(t)=\exp (t H)$ задает однопараметрическую подгруппу в $U$. Кривая $\gamma(t)=R(t) O$ будет натурально параметризованной геодезической в $M[10$, гл. IV, теорема 3.3]. Так как все геодезические на $M$ замкнуты и имеют длину $2 \pi$, то $R(t+2 \pi) O=R(t) O$, в частности, $R(2 \pi) O=O$, следовательно, $R(2 \pi) \in K$.

Пусть $d u$ и $d k$ - элементы нормированных единищей мер Хаара на компактных группах $U$ и $K$, соответственно.

ЛЕмма 2.1. Если $x=v O, v \in U, m o$

$$
S_{t} f(x)=\int_{K} f(v k R(t) O) d k .
$$

ДокАЗАТЕЛЬСТво. Временно обозначим

$$
\widetilde{S}_{t} f(x)=\int_{K} f(v k R(t) O) d k, \quad x=v O
$$

Для любой функции $f(x)$ на $M$ и $u \in U$ пусть

$$
f^{u}(x)=f(u x)
$$

Очевидно, что $\left(S_{t} f\right)^{u}=S_{t}\left(f^{u}\right)$ и $\left(\widetilde{S}_{t} f\right)^{u}=\widetilde{S}_{t}\left(f^{u}\right)$, поэтому для доказательства того, что

$$
S_{t} f(x)=\widetilde{S}_{t} f(x) \quad \forall f \in L_{p}(M)
$$

достаточно показать, что $S_{t} f(O)=\widetilde{S}_{t} f(O)$ для любой непрерывной функции $f(x)$ на $M$ (так как непрерывные функции образуют плотное подмножество в $L_{p}(M)$ ). 
Для любой непрерывной функции $h(x)$ на сфере $S_{M}(O ; t)$ определим функционалы

$$
I_{1}(h)=\left(S_{t} h\right)(O) \text { и } I_{2}(h)=\left(\widetilde{S}_{t} h\right)(O) .
$$

Очевидно, что $I_{1}$ и $I_{2}$ линейные положительные функционалы, инвариантные относительно действия группы $K$, т.е.

$$
I_{1}\left(h^{k}\right)=I_{1}(h), \quad I_{2}\left(h^{k}\right)=I_{2}(h) \quad \forall k \in K .
$$

Следовательно, функционалы $I_{1}$ и $I_{2}$ определяют $K$-инвариантные меры на сфере $S_{M}(O ; t)$. Так как $S_{M}(O ; t)$ - однородное пространство для группы $K$, то инвариантная мера единственна с точностью до множителя [11], а так как для функции $h_{0}(x) \equiv 1$ будет

$$
I_{1}\left(h_{0}\right)=I_{2}\left(h_{0}\right)=1
$$

то функционалы $I_{1}$ и $I_{2}$ совпадают.

ЗАмечание. Оператор сдвига $S_{t} f$ можно также записать в виде

$$
S_{t} f(x)=\frac{1}{\sigma} \int_{S(x)} f(\gamma(x, \xi ; t)) d \mu(\xi)
$$

где $\sigma, \gamma(x, \xi ; t), S(x)$ определены в $\S 1$. Доказательство того, что формулы (2.4) и (2.3) задают один и тот же оператор проводится, как в лемме 2.1. Формула (2.4) позволяет распространить функцию $S_{t} f(x)$ на все значения $t \in \mathbb{R}$, причем эта функция будет четной. Отметим также, что разность ${ }^{*} \Delta_{t}^{k} f(x)$ можно записать в виде

$$
{ }^{*} \Delta_{t}^{k} f(x)=\left.\Delta_{t}^{k} S_{\tau} f(x)\right|_{\tau=0}
$$

где

$$
\Delta_{t}^{k} S_{\tau} f(x)=\sum_{j=0}^{k}(-1)^{k-j} S_{\tau+j t} f(x)
$$

- обычная $k$-я разность с шагом $t$ по переменной $\tau$.

Определим функцию

$$
\alpha(t)=\left(\sin \frac{t}{2}\right)^{a}(\sin t)^{b},
$$

где числа $a$ и $b$ берутся из (1.2).

Пусть $F(u)$ - интегрируемая по мере Хаара функция на группе $U$. Любой элемент $u \in U$ можно представить в виде $u=k_{1} R(t) k_{2}$, где $k_{1}, k_{2} \in K, 0 \leqslant t \leqslant \pi$. Тогда справедлива следующая формула $[10$, гл. Х, $\S 1]$, которая неоднократно будет использоваться в дальнейшем

$$
\int_{U} f(u) d u=A \int_{0}^{\pi} \int_{K \times K} F\left(k_{1} R(t) k_{2}\right) \alpha(t) d k_{1} d k_{2},
$$

где

$$
A=\left(\int_{0}^{\pi} \alpha(t) d t\right)^{-1}
$$


Из единственности (с точностью до множителя) инвариантной меры на $M$ следует, что сушествует постоянная $C>0$, для которой

$$
\int_{M} f(x) d x=C \int_{U} f(u O) d u
$$

для любой интегрируемой функции $f(x)$ на $M$. Из (2.8) и (2.9) следует, что

$$
\int_{M} f(x) d x=A_{1} \int_{0}^{\pi} \int_{K} f(k R(t) O) \alpha(t) d t d k,
$$

где $A_{1}=A C$. В частности, если $f$ зависит только от расстояния от некоторой фиксированной точки $x_{0} \in M$ до точки $x$ (т.е. $f(x)=\widetilde{f}\left(\left|x_{0}, x\right|\right)$, без ограничения общности можно считать, что $\left.x_{0}=O\right)$, то

$$
\int_{M} f(x) d x=A_{1} \int_{0}^{\pi} \widetilde{f}(t) \alpha(t) d t
$$

Лемма 2.2. Пусть $T(u)$ - сферическое представление группы $U, \varphi(u)-$ соответствующая ему сферическая функция, $f(x) \in L_{p}(M)$. Тогда функция

$$
\Phi(x)=\int_{0}^{\pi} \varphi(R(t)) S_{t} f(t) \alpha(t) d t
$$

будет сферическим полиномом.

ДокАЗАТЕЛЬСтво. Пусть $V-$ пространство представления $T, d=\operatorname{dim} V$, $\langle\cdot, \cdot\rangle$ - инвариантная эрмитова форма, $e_{i}(0 \leqslant i \leqslant d-1)$ - ортонормированный базис в $V$, причем вектор $e_{0}-K$-инвариантный, $\tau_{i j}(u)$ - матричные элементы представления $T(u)$. Тогда $\varphi(u)=\tau_{00}(u)$.

Рассмотрим сферический полином на $M$

$$
\Phi(x)=\Phi(v O)=\sum_{i=0}^{d-1} c_{i} \tau_{i 0}(v), \quad v \in U, \quad x=v O \in M,
$$

где

$$
c_{i}=A^{-1} \int_{U} f(u O) \bar{\tau}_{i 0}(u) d u,
$$

$A$ - коэффициент из (2.8). Пользуясь унитарностью представления $T$ и инвариантностью меры Хаара получим, что

$$
\begin{aligned}
\Phi(v O) & =\sum_{i} A^{-1} \int_{U} f(u O) \tau_{0 i}\left(u^{-1}\right) \tau_{i 0}(v) d u \\
& =A^{-1} \int_{U} f(u O) \tau_{00}\left(u^{-1} v\right) d u=A^{-1} \int_{U} f(v u O) \tau_{00}\left(u^{-1}\right) d u .
\end{aligned}
$$

Так как $\tau_{00}\left(k_{1} u k_{2}\right)=\tau_{00}(u) \forall k_{1}, k_{2} \in K$ и $\tau_{00}\left(u^{-1}\right)=\tau_{00}(u)$, то из $(2.13)$, используя $(2.8)$ и лемму 2.1 , получим

$$
\begin{aligned}
\Phi(v O) & =A^{-1} \int_{U} f(v u O) \tau_{00}(u) d u \\
& =\int_{0}^{\pi} \int_{K} f(v k R(t) O) \tau_{00}(R(t)) \alpha(t) d k d t=\int_{0}^{\pi} \varphi(R(t)) S_{t} f(x) \alpha(t) d t .
\end{aligned}
$$


ЛЕмма 2.3. Пусть $J(t)$ - произвольный четный тригонометрический полином степени т. Тогда функция

$$
\Phi(x)=\int_{0}^{\pi} J(t) S_{t} f(x) \alpha(t) d t
$$

есть сферический полином степени т.

ДоказАТЕЛЬСтво. Известна явная формула для сферической функции $\varphi^{l}(u)$ представления $T^{l}$. Из [9, гл. V, $\S 4$, п. 3] получаем, что

$$
\varphi^{l}(R(t))=F\left(\frac{1}{2} a+b+l,-l ; \frac{1}{2}(a+b+1) ; \sin ^{2} \frac{t}{2}\right),
$$

где $F(a, b, c ; z)$ - гипергеометрическая функция, причем при наших значениях параметров она сводится к полиному Якоби (см. [12, т. 2, формула (10.8.16)]):

$$
\varphi^{l}(R(t))=\frac{l ! \Gamma(\alpha+1)}{\Gamma(l+\alpha+1)} P_{l}^{(\alpha, \beta)}(\cos t)
$$

где $P_{l}^{(\alpha, \beta)}(x)$ - полином Якоби степени $l, \alpha=\frac{1}{2}(a+b-1), \beta=\frac{1}{2}(b-1)$. В частности, $\varphi^{l}(R(t))$ - четньй тригонометрический полином степени $l$.

Любой четный тригонометрический полином $J(t)$ будет линейной комбинацией полиномов $\varphi^{l}(R(t)), l=0,1, \ldots m$, и далее лемма 2.3 следует из леммы 2.2 .

Мы считаем, что $M \neq S^{n}$, поэтому из (1.2) следует, что $a$ - четное, а $b$ - нечетное натуральные числа. Как и в [3], определим функцию $S_{t}^{-} f(x)$ как нечетное $2 \pi$-периодическое продолжение функции $S_{t} f(x)$ с промежутка $0 \leqslant t \leqslant \pi$ ( $f$ и $x$ фиксированы) на все значения $t \in \mathbb{R}$, т.е.

$$
S_{t}^{-} f(x)=(-1)^{[t / \pi]} S_{t} f(x)
$$

где $[a]$ - целая часть числа $a$. Отметим, что из (2.2) следует, что

$$
\left\|S_{t}^{-} f(x)\right\|_{p} \leqslant\|f\|_{p}
$$

Лемма 2.4. Пусть $J(t)$ - четный тригонометрический полином степени $m, j$ - натуральное число. Тогда функиия

$$
\Phi_{j}(x)=\int_{0}^{\pi} J(t) S_{j t}^{-} f(x) \alpha(j t) d t
$$

есть сферический полином степени $m$. 
ДоказАТеЛЬство. а) Пусть $\varphi(t)=\left(S_{t}^{-} f(x)\right) \alpha(t)$. Тогда $\varphi(t)$-четная $2 \pi$-периодическая функция. Пусть $\nu$ - натуральное число. Проверим, что

$$
\int_{0}^{\pi} \varphi(j t) \cos \nu t d t=\frac{1}{2} \int_{-\pi}^{\pi} \varphi(j t) \cos \nu t d t=0,
$$

если число $\nu / j$ не целое.

Пусть

$$
I=\int_{-\pi}^{\pi} \varphi(j t) \cos \nu t d t .
$$

Сделаем замену переменной $t=s+\frac{2 \pi}{j}$ и воспользуемся тем, что функция $\varphi(j t) \cos \nu t-2 \pi$-периодическая. Тогда

$$
\begin{aligned}
I & =\int_{-\pi}^{\pi} \varphi(j s) \cos \left(\nu s+\frac{2 \pi \nu}{j}\right) d s \\
& =\cos \frac{2 \pi \nu}{j} \int_{-\pi}^{\pi} \varphi(j s) \cos \nu s d s-\sin \frac{2 \pi \nu}{j} \int_{-\pi}^{\pi} \varphi(j s) \sin \nu s d s=I \cos 2 \pi \frac{\nu}{j} .
\end{aligned}
$$

Следовательно, $I\left(1-\cos 2 \pi \frac{\nu}{j}\right)=0$ и $I=0$, если $\nu / j$ не целое.

б) Пусть

$$
J(t)=\sum_{0 \leqslant \nu \leqslant m} c_{\nu} \cos \nu t .
$$

Обозначим через $\widetilde{J}(j t)$ тригонометрический полином, который получится, если в (2.15) оставить только слагаемые, у которых $\nu$ кратно $j$. Из а) следует, что

$$
\Phi_{j}(x)=\int_{0}^{\pi} \widetilde{J}(j t) \varphi(j t) d t .
$$

Если сделать замену переменной $s=j t$ и воспользоваться тем, что $\widetilde{J}(s)$ и $\varphi(s)-$ четные $2 \pi$-периодические функции, то легко получить, что

$$
\Phi_{j}(x)=\int_{0}^{\pi} \widetilde{J}(s) \varphi(s) d s,
$$

но последнее выражение является сферическим полиномом по лемме 3.

Пусть

$$
\mathscr{D}_{m}(u)=\left(\frac{\sin \frac{m u}{2}}{\sin \frac{u}{2}}\right)^{2 s}, \quad m, s=1,2, \ldots,
$$

- ядро Джексона, являюшееся четным тригонометрическим полиномом степени $s(m-1)$. Положим (с учетом $(2.11))$

$$
\begin{gathered}
\Omega_{m}=\int_{M} \mathscr{D}_{m}\left(\left|x_{0}, x\right|\right) d x=A_{1} \int_{0}^{\pi} \mathscr{D}_{m}(t) \alpha(t) d t ; \\
\mathscr{I}_{m}(u)=\frac{A_{1}}{\Omega_{m}} \mathscr{D}_{m}(u) .
\end{gathered}
$$

Отметим, что

$$
\int_{0}^{\pi} \mathscr{I}_{m}(u) \alpha(u) d u=1
$$


ЛЕмма 2.5. При фиксированном $s$ u $m \rightarrow \infty$ справедливь следующие асимптотические равенства

$$
\begin{aligned}
\Omega_{m} \sim m^{2 s-a-b-1}, & 2 s-a-b-1>0 \\
\int_{0}^{\pi} t^{r} \mathscr{I}_{m}(t) \alpha(t) d t \sim m^{-r}, & 2 s-a-b-1>r>0
\end{aligned}
$$

где, как обычно, $A_{m} \sim B_{m}$, если $A_{m}, B_{m}>0$, и $c_{1} B_{m} \leqslant A_{m} \leqslant c_{2} B_{m}$ для некоторых констант $c_{1}, c_{2}>0$.

ДокАЗАТЕЛЬСтво. Воспользовавшись неравенствами

$$
\left|\frac{\sin x}{x}\right| \leqslant 1 \quad(x \in \mathbb{R}), \quad(\sin x)^{-1} \leqslant \frac{\pi}{2} x^{-1} \quad\left(0<x<\frac{\pi}{2}\right),
$$

получаем следуюшую оценку сверху

$$
\begin{aligned}
\Omega_{m} & =A_{1} \int_{0}^{\pi}\left(\sin \frac{m t}{2}\right)^{2 s} 2^{b}\left(\sin \frac{t}{2}\right)^{a+b-2 s}\left(\cos \frac{t}{2}\right)^{b} d t \\
& \leqslant c_{1} \int_{0}^{\pi}\left(\sin \frac{m t}{2}\right)^{2 s} t^{a+b-2 s} d t=c_{1} m^{2 s-a-b-1} \int_{0}^{m \pi}\left(\sin \frac{u}{2}\right)^{2 s} u^{a+b-2 s} d u \\
& \leqslant m^{2 s-a-b-1} c_{1} \int_{0}^{\infty}\left(\sin \frac{u}{2}\right)^{2 s} u^{a+b-2 s} d u=c_{2} m^{2 s-a-b-1}
\end{aligned}
$$

При этом сделана замена $u=m t ; c_{1}, c_{2}$ (и далее $c_{3}, c_{4}$ ) - не зависящие от $m$ постоянные.

В другую сторону,

$$
\begin{aligned}
\Omega_{m} & \geqslant A_{1} \int_{0}^{\pi / 2}\left(\sin \frac{m t}{2}\right)^{2 s} 2^{b}\left(\sin \frac{t}{2}\right)^{a+b-2 s}\left(\cos \frac{t}{2}\right)^{b} d t \\
& \geqslant c_{3} \int_{0}^{\pi / 2}\left(\sin \frac{m t}{2}\right)^{2 s} t^{a+b-2 s} d t \\
& =c_{3} m^{2 s-a-b-1} \int_{0}^{m \pi / 2}\left(\sin \frac{u}{2}\right)^{2 s} u^{a+b-2 s} d u \geqslant c_{4} m^{2 s-a-b-1},
\end{aligned}
$$

что доказывает (2.17).

Такими же оценками показьваем, что

$$
p_{m}=\int_{0}^{\pi} t^{r}\left(\frac{\sin \frac{m t}{2}}{\sin \frac{t}{2}}\right)^{2 s} \alpha(t) d t \sim m^{2 s-a-b-r-1} .
$$

Тогда

$$
\int_{0}^{\pi} t^{r} \mathscr{I}_{m}(t) \alpha(t) d t \sim \frac{p_{m}}{\Omega_{m}} \sim m^{-r}
$$


ЛЕмма 2.6. Если число $m$ делится на $2 j$, то функиия

$$
\Phi_{m, j}(x)=\int_{0}^{\pi} \mathscr{I}_{m}(t) S_{j t}^{-} f(x) \alpha(t) d t
$$

будет сферическим полиномом степени $m(s-1)$.

ДокАЗАТЕЛЬСтво. Воспользуемся тождеством

$$
\begin{aligned}
\mathscr{I}_{m}(t) \alpha(t)=\frac{A_{1}}{\Omega_{m}} 2^{a+b}\left(\frac{\sin \frac{m t}{2}}{\sin j t}\right)^{2 s} & \left(\frac{\sin j t}{\sin \frac{t}{2}}\right)^{2 s-a-b-1} \\
& \times\left(\cos \frac{j t}{2}\right)^{a}\left(\cos \frac{t}{2}\right)^{b-1}\left(\frac{\sin j t \cos \frac{t}{2}}{\sin \frac{t}{2}}\right) \alpha(j t) .
\end{aligned}
$$

Легко видеть, что каждый из множителей, кроме $\alpha(j t)$, является четным тригонометрическим полиномом (так как $m$ делится на $2 j$, число $a$ - четное, число $b$ - нечетное). Остается воспользоваться леммой 2.4 и тем, что

$$
\operatorname{deg} \frac{\mathscr{I}_{m}(t) \alpha(t)}{\alpha(j t)}=(m-1) s+\left(\frac{a}{2}+b\right)(1-s) \leqslant(m-1) s
$$

Доказательство следующей леммы и прямой части теоремы 1 проводится аналогично доказательству леммы 2.2 и прямой части теоремы 1.2 в [3].

Лемма 2.7. Если $f \in L^{p}(M), 1 \leqslant p \leqslant \infty$, и m делится на $2(k !)$, то существует сферический полином $\Phi_{N}(x)$ степени $N=s(m-1)$, для которого

$$
f(x)-\Phi_{N}(x)=\left.(-1)^{k} \int_{0}^{\pi} \Delta_{t}^{k} S_{\tau}^{-} f(x)\right|_{\tau=0} \mathscr{I}_{m}(t) \alpha(t) d t
$$

гдe

$$
\Delta_{t}^{k} S_{\tau}^{-} f(x)=\sum_{j=0}^{k}(-1)^{k-j} C_{k}^{j} S_{\tau+j t}^{-} f(x) .
$$

ДокАЗАТЕЛЬСтво. Правую часть в (2.20) можно записать в виде (см. (2.16), $(2.19))$

$$
\begin{aligned}
f(x)+\sum_{j=1}^{k}(-1)^{j} C_{k}^{j} \int_{0}^{\pi} S_{j t}^{-} f(x) \mathscr{I}_{m}(t) \alpha(t) d t & \\
& =f(x)+\sum_{j=1}^{k}(-1)^{j} C_{k}^{j} \Phi_{N, j}(x)=f(x)-\Phi_{N}(x),
\end{aligned}
$$

где $\Phi_{N, j}(x)$, а, следовательно, и $\Phi_{N}(x)$ - сферические полиномы по лемме 2.7 . 
ДОКАЗАТЕЛЬСТВО ПРЯМОЙ ЧАСТИ ТЕОРЕМЫ 1 . Пусть $f \in L_{p}(M)$ и вЫПоЛняется условие

$$
\left\|^{*} \Delta_{t}^{k} f(x)\right\|_{p} \leqslant c_{f} t^{r}, \quad k>r>0
$$

Пусть $N$ - целое число вида $N=s(m-1)$ и $m$ делится на $2(k !)$. Из леммы 2.7 следует, что

$$
\begin{aligned}
\left|f(x)-\Phi_{N}(x)\right| \leqslant\left|\int_{0}^{\pi / k} \Delta_{t}^{k} S_{\tau} f(x)\right|_{\tau=0} \mathscr{I}_{m}(t) \alpha(t) d t \mid & \\
& +\left|\sum_{j=0}^{k}(-1)^{k-j} C_{k}^{j} \int_{\pi / k}^{\pi} S_{j t}^{-} f(x) \mathscr{I}_{m}(t) \alpha(t) d t\right| .
\end{aligned}
$$

В первом слагаемом вместо $S_{\tau}^{-}$стоит $S_{\tau}$, так как при $t \in[0, \pi / k]$ будет $j t \in[0, \pi]$ и $S_{j t}^{-}=S_{j t}$.

Далее получаем, что

$$
\begin{aligned}
& \left\|f(x)-\Phi_{N}(x)\right\|_{p} \\
& \quad \leqslant \int_{0}^{\pi / k}\left\|^{*} \Delta_{t}^{k} f(x)\right\|_{p} \mathscr{I}_{m}(t) \alpha(t) d t+\sum_{j=0}^{k} C_{k}^{j} \int_{\pi / k}^{\pi}\left\|S_{j t}^{-} f(x)\right\|_{p} \mathscr{I}_{m}(t) \alpha(t) d t \\
& \quad \leqslant C_{f} \int_{0}^{\pi / k} t^{r} \mathscr{I}_{m}(t) \alpha(t) d t+2^{k}\|f\|_{p} \frac{A_{1}}{\Omega_{m}} \int_{\pi / k}^{\pi} \mathscr{D}_{m}(t) \alpha(t) d t \\
& \quad \leqslant c_{1}\left(\frac{c_{f}}{N^{r}}+\frac{\|f\|_{p}}{N^{2 s-a-b-1}}\right) \leqslant c_{2} \frac{\left(c_{f}+\|f\|_{p}\right)}{N^{r}}=c_{2} \frac{\|f\|_{H_{p}^{r}(M)}}{N^{r}}
\end{aligned}
$$

если $2 s-a-b-1>r$. При этом использовано условие (2.21), оценки (2.17), (2.18) и то, что функция $\mathscr{D}_{m}(t)$ на отрезке $[\pi / k, \pi]$ ограничена.

Получена оценка

$$
\left\|f-\Phi_{N}\right\|_{p} \leqslant c_{2} \frac{\|f\|_{H_{p}^{r}(M)}}{N^{r}}
$$

Если $N \geqslant s(2(k !)-1)$ - произвольное натуральное число, то подбираем $m$ такое, что $m$ делится на $2(k !)$ и $s(m-1) \leqslant N<s m$. Пусть $\Phi_{N}=\Phi_{s(m-1)}-$ полином степени $N$, определяемый из (2.20). Мы получили последовательность сферических полиномов $\Phi_{N}$, для которых

$$
E_{N}(f)_{p} \leqslant\left\|f-\Phi_{N}\right\|_{p} \leqslant c_{2} \frac{\|f\|_{H_{p}^{r}(M)}}{(s(m-1))^{r}} \leqslant c_{3} \frac{\|f\|_{H_{p}^{r}(M)}}{N^{r}}
$$




\section{§3. Неравенства типа Бернштейна}

Лемма 3.1. Пусть $\Phi(x)$ - сферический полином степени $m$. Тогда функиия $\rho(t)=\Phi(R(t) O)$ является тригонометрическим полиномом степени $\mathrm{m}$.

ДоКАЗАТЕЛЬСТво. Любой сферический полином есть линейная комбинация матричных элементов $\tau_{j 0}^{l}(u)$ сферических представлений $T^{l}$ с $l \leqslant m$, поэтому достаточно показать, что $\tau_{j 0}^{l}(R(t))$ - тригонометрический полином степени $l$.

Воспользуемся обозначениями из $\S 1$. Пусть $T^{l}(u)$ - сферическое представление групшы $U$ со старшим весом $\lambda=l \mu, E^{l}$ - пространство этого представления, $e_{j}^{l}$ $\left(0 \leqslant j \leqslant d_{l}-1\right)$ - ортонормированный базис пространства $E^{l}$, причем вектор $e_{0}^{l}-$ $K$-инвариантный.

Векторные пространства $\mathfrak{u}, \mathfrak{k}, \mathfrak{a}, \mathfrak{h}$ определены в $\S 1$. Пусть $\mathfrak{u}_{\mathbb{C}}, \mathfrak{k}_{\mathbb{C}}, \mathfrak{a}_{\mathbb{C}}$, и $\mathfrak{h}_{\mathbb{C}}-$ комплексификации этих пространств. Тогда $\mathfrak{u}_{\mathbb{C}}$ будет полупростой алгеброй Ли, $\mathfrak{h}_{\mathbb{C}}$ - подалгебра Картана и можно пользоваться методами теории полупростых алгебр Ли (см., например, [13], [14]). Веса и корни алгебры $\mathfrak{u}_{\mathbb{C}}$ являются линейными функционалами на $\mathfrak{h}_{\mathbb{C}}$ и принимают вещественные значения на вещественном векторном пространстве $\mathfrak{h}_{\mathbb{R}}=i \mathfrak{h}, i=\sqrt{-1}$. Выберем какой-нибудь базис $h_{1}, h_{2}, \ldots$ в $i \mathfrak{h}$, причем пусть $h_{1} \in \mathfrak{a}$. Обычным образом по этому базису строится упорядочение на сопряженном пространстве $\mathfrak{h}_{\mathbb{R}}^{*}$. Пусть $\Sigma^{+}-$множество положительных корней алгебры $\mathfrak{u}_{\mathbb{C}}$.

Напомним, что $H \in \mathfrak{a},|H|=1$ и $R(t)=\exp (t H)$. Пусть $\delta$-какой-нибудь вес представления $T^{l}$. Любой вес имеет вид

$$
\delta=\lambda-\sum_{\alpha \in \Sigma^{+}} n_{\alpha} \cdot \alpha
$$

где $n_{\alpha}$ - неотрицательные целые числа, $\lambda=l \mu-$ старший вес. Известно (см. [9, гл. V, §4, п. 3]), что для симметрического пространства ранга 1 ограничение любого положительного корня $\alpha$ на $\mathfrak{a}$ равно

$$
\left.\alpha\right|_{\mathfrak{a}}=\left.k \mu\right|_{\mathfrak{a}},
$$

где число $k$ может быть равно $0, \frac{1}{2}$ или 1 . Тогда

$$
\delta(H)=q \cdot \mu(H),
$$

где $q$ - целое или полуцелое неотрицательное число и $q \leqslant l$. При выбранной нормировке $\mu(H)=2 i$, следовательно,

$$
\delta(H)=2 q i, \quad q \leqslant l .
$$

Так как сферическое представление эквивалентно своему дуальному представлению, то вместе с весом $\delta$ весом будет также и $(-\delta)$ (см. [14, гл. VII, $\S 7$, п. 5]), тогда

$$
(-\delta)(H)=2(-q) i \text { и }(-q) \leqslant l .
$$


Следовательно, $|q| \leqslant l$.

В пространстве $E^{l}$ можно выбрать базис из весовых векторов. В этом базисе матрица оператора $T^{l}(R(t))$ будет диагональной, с диагональньги элементами вида $e^{i q t}$, где $q$ - неотрицательные целые или полуцелые числа и $|q| \leqslant l$. В любом другом базисе матричные элементы $\tau_{j 0}^{l}(R(t))$ будут иметь вид

$$
\tau_{j 0}^{l}(R(t))=\sum_{q} c_{q} e^{i q t}
$$

где $c_{q}$ - числовые коэффициенты. Так как $R(2 \pi) \in K($ см. § 2$)$, то

$$
\tau_{j 0}^{l}(R(t+2 \pi))=\tau_{j 0}^{l}(R(t) R(2 \pi))=\tau_{j 0}^{l}(R(t)),
$$

следовательно, в сумме (3.1) коэффициенты при полуцелых $q$ равны 0, и значит, $\tau_{j 0}^{l}(R(t))$ - тригонометрический полином степени $l$.

Пусть $x, x^{\prime} \in M$ и $\xi, \xi^{\prime}$-произвольные единичные касательные векторы в точках $x$ и $x^{\prime}$, соответственно. Так как $M-$ КРОСП, то известно [ $\left.15, \S 8.12\right]$, что существует изометрия $g \in U$, которая переводит точку $x$ в $x^{\prime}$ и вектор $\xi$ в $\xi^{\prime}$ (точнее говоря, $g_{*}(\xi)=\xi^{\prime}$, где $g_{*}$ - индуцированное отображение касательных векторов). В частности, если $\xi, \xi^{\prime} \in T_{0} M$, то существует изометрия $k \in K$, для которой $k_{*}(\xi)=\xi^{\prime}$.

Пусть $x \in M, \xi$ - единичный касательньй вектор в точке $x, \gamma(x, \xi ; s)$ - геодезическая, выходящая из точки $x$ в направлении вектора $\xi$. Для $t \in \mathbb{R}$ и натурального $k$ положим

$$
{ }_{t} \partial_{\xi}^{k} f(x)=\left.\frac{d^{k}}{d s^{k}} f(\gamma(x, \xi ; s))\right|_{s=t} .
$$

Лемма 3.2. Пусть $\Phi(x)$ - сферический полином степени $m$. Тогда выполняется неравенство

$$
\left|{ }_{t} \partial_{\xi}^{k} \Phi(x)\right| \leqslant m^{k}\|\Phi\|_{\infty}
$$

ДокаЗАТЕЛЬство. Пусть $g$ - изометрия, переводящая точку $O$ в точку $x$ и вектор $H \in \mathfrak{p}$ (напомним, что касательное пространство $T_{O} M$ отождествляется $c \mathfrak{p})$ в вектор $\xi$. Заменив полином $\Phi(x)$ на полином $\widetilde{\Phi}(x)=\Phi(g x)$, мы получим, что неравенство (3.3) достаточно доказать для случая, когда $x=O$ и $\xi=H$. Тогда $\gamma(O, H ; s)=R(s) O$ и

$$
{ }_{t} \partial_{H}^{k} \Phi(O)=\frac{d^{k}}{d t^{k}} \Phi(R(t))
$$

Но по лемме $3.1 \Phi(R(t))$ - тригонометрический полином степени $m$ и остается воспользоваться классическим неравенством Бернштейна для тригонометрических полиномов:

$$
\left|\frac{d^{k}}{d t^{k}} \Phi(R(t))\right| \leqslant m^{k} \max _{0 \leqslant t \leqslant 2 \pi}|\Phi(R(t))| \leqslant m^{k} \max _{x \in M}|\Phi(x)| .
$$


Пусть $B$ - многообразие единичных касательных векторов к многообразию $M$. Точки многообразия $B$ имеют вид $(x, \xi)$, где $x \in M, \xi \in S(x)$. Для любой непрерывной функции $F(x, \xi)$ на $B$ можно определить инвариантный (относительно группы $U$ ) интеграл формулой

$$
I(F)=\int_{M} d x \int_{S(x)} F(x, \xi) d \mu(\xi),
$$

где $d \mu(\xi)$ - элемент римановой меры на сфере $S(x)$. Из единственности (с точностью до множителя) инвариантного интеграла следует, что его можно также записать в виде

$$
I(F)=A_{3} \int_{U} F\left(u x_{0}, u_{*}\left(\xi_{0}\right)\right) d u,
$$

где $d u$ - элемент меры Хаара на группе $U,\left(x_{0}, \xi_{0}\right)$ - любая фиксированная точка из $B, A_{3}$ - не зависящая от $F$ константа.

Для любой функции $F(x, \xi)$ на $B$ определим "усредненную” функцию $F_{0}(x, \xi)$ формулой

$$
F_{0}(x, \xi)=\frac{1}{2 \pi} \int_{0}^{2 \pi} F\left(\gamma(x, \xi ; s), \frac{d}{d s} \gamma(x, \xi ; s)\right) d s .
$$

Лемма 3.3. Если $F(x, \xi)$ - непрерывная функция на $B$, mo

$$
I(F)=I\left(F_{0}\right)
$$

ДоКАЗАТЕЛЬСТво. Пусть $v$-изометрия из группы $U$, которая переводит точку $O$ в точку $x$ и вектор $H \in T_{O}=\mathfrak{p}$ в вектор $\xi$. Тогда $\gamma(x, \xi ; s)=v R(s) O$ и $\frac{d}{d s} \gamma(x, \xi ; s)=(v R(s))_{*} H$. Следовательно,

$$
F_{0}(x, \xi)=F_{0}\left(v O, v_{*} H\right)=\frac{1}{2 \pi} \int_{0}^{2 \pi} F\left(v R(s) O,(v R(s))_{*} H\right) d s
$$

Пользуясь (3.5), получим, что

$$
\begin{aligned}
I_{0}(F) & =A_{3} \int_{U} F_{0}\left(u O, u_{*} H\right) d u=\frac{A_{3}}{2 \pi} \int_{0}^{2 \pi} \int_{U} F\left(u R(s) O,(u R(s))_{*} H\right) d u d s \\
& =\frac{A_{3}}{2 \pi} \int_{0}^{2 \pi} \int_{U} F\left(\widetilde{u} O, \widetilde{u}_{*} H\right) d \widetilde{u} d s=A_{3} \int_{U} F\left(\widetilde{u} O, \widetilde{u}_{*} H\right) d \widetilde{u}=I(F) .
\end{aligned}
$$

При этом была сделана замена $\widetilde{u}=u R(s)$ и использована инвариантность меры Хаара относительно правых сдвигов.

Исходя из оператора ${ }_{t} \partial_{\xi}^{k} f(x)$ (см. (3.2)), определим усредненный оператор ${ }_{t} \partial^{k} f(x)$ как

$$
{ }_{t} \partial^{k} f(x)=\frac{1}{\sigma} \int_{S(x)}{ }_{t} \partial_{\xi}^{k} f(x) d \mu(\xi) .
$$


ЛЕмма 3.4. Пусть $\Phi(x)$ - сферический полином порядка $m$. Тогда справедливо неравенство

$$
\left\|_{t} \partial^{k} \Phi(x)\right\|_{p} \leqslant c m^{k}\|\Phi\|_{p}, \quad 1 \leqslant p \leqslant \infty
$$

әде с - не зависящая от $m, \Phi, k$ постоянная.

ДокАЗАТЕЛЬСтво. При $p=\infty$ неравенство сразу следует из леммы 3.2 . Пусть $p<\infty$. Пользуясь неравенством Гёльдера, получим, что

$$
\begin{aligned}
\left\|_{t} \partial^{k} \Phi(x)\right\|_{p}^{p} & =\int_{M}\left|\int_{S(x)}{ }_{t} \partial_{\xi}^{k} \Phi(x) d\left(\frac{\mu(\xi)}{\sigma}\right)\right|^{p} d x \\
& \leqslant \int_{M} \int_{S(x)}\left|{ }_{t} \partial_{\xi}^{k} \Phi(x)\right|^{p} d\left(\frac{\mu(\xi)}{\sigma}\right) d x=\frac{1}{\sigma} I\left(\left.{ }_{t} \partial_{\xi}^{k} \Phi(x)\right|^{p}\right) .
\end{aligned}
$$
Пусть $F(x, \xi)=\left|{ }_{t} \partial_{\xi}^{k} \Phi(x)\right|^{p}=\left|\frac{d^{k}}{d t^{k}} \Phi(\gamma(x, \xi ; t))\right|^{p}, t-$ фиксированное число. Тогда
очевидно, что

$$
F\left(\gamma(x, \xi ; s), \frac{d}{d s} \gamma(x, \xi ; s)\right)=\left|\frac{d^{k}}{d t^{k}} \Phi(\gamma(x, \xi ; t+s))\right|^{p}
$$

В обозначениях леммы 3.3

$$
\begin{aligned}
F(x, \xi) & =\left|\frac{d^{k}}{d t^{k}} \Phi(v R(t) O)\right|^{p}, \\
F_{0}(x, \xi) & =\frac{1}{2 \pi} \int_{0}^{2 \pi}\left|\frac{d^{k}}{d t^{k}} \Phi(v R(t+s) O)\right|^{p} d t=\frac{1}{2 \pi} \int_{0}^{2 \pi}\left|\frac{d^{k}}{d t^{k}} \Phi(v R(t) O)\right|^{p} d t .
\end{aligned}
$$

Так как $\Phi(v R(t) O)$ - тригонометрический полином порядка $m$, то из обычного неравенства Бернштейна в метрике $L_{p}^{*}($ см. $[16, \S 2.5])$ следует, что

$$
F_{0}(x, \xi) \leqslant \frac{1}{2 \pi} m^{p k} \int_{0}^{2 \pi}|\Phi(v R(t) O)|^{p} d t .
$$

Применяя лемму 3.3 , получим

$$
\begin{aligned}
I(F) & =I\left(F_{0}\right) \leqslant m^{p k} \frac{1}{2 \pi} I\left(\int_{0}^{2 \pi}|\Phi(v R(t) O)|^{p} d t\right) \\
& =\frac{m^{p k}}{2 \pi} \int_{0}^{2 \pi} \int_{U}|\Phi(u R(t) O)|^{p} d u d t=m^{p k} \int_{U}|\Phi(\widetilde{u} O)|^{p} d \widetilde{u}=\frac{m^{p k}}{A_{3}}\|\Phi\|_{p}^{p} .
\end{aligned}
$$

Окончательно получаем, что

$$
\left\|_{t} \partial^{k} \Phi\right\|_{p} \leqslant c m^{k}\|\Phi\|,
$$

где $c=\left(\sigma A_{3}\right)^{-1 / p}$. 
ЛЕмма 3.5. Если $\Phi(x)$ - сферический полином порядка $m$, то

$$
\left\|^{*} \Delta_{t}^{k} \Phi(x)\right\|_{p} \leqslant c(m t)^{k}\|\Phi\|_{p}, \quad 1 \leqslant p \leqslant \infty
$$

где постоянная с не зависит от $\Phi, m, t(t>0)$.

ДоказАТЕЛЬство. Разность

$$
\left.\Delta_{t}^{k} \Phi(\gamma(x, \xi ; s))\right|_{s=0}
$$

допускает представление в виде

$$
\left.\int_{0}^{t} \cdots \int_{0}^{t} \frac{d^{k}}{d \tau^{k}} \Phi(\gamma(x, \xi ; \tau))\right|_{\tau=t_{1}+\cdots+t_{k}} d t_{1} \ldots d t_{k}
$$

Тогда

$$
{ }^{*} \Delta_{t}^{k} \Phi(x)=\int_{0}^{t} \cdots \int_{0}^{t}\left(t_{1}+\cdots+t_{k}\right) \partial^{k} \Phi(x) d t_{1} \ldots d t_{k} .
$$

Пользуясь леммой 3.4 , получаем, что

$$
\left\|^{*} \Delta_{t}^{k} \Phi(x)\right\|_{p} \leqslant \int_{0}^{t} \cdots \int_{0}^{t}\left\|_{\left(t_{1}+\cdots+t_{k}\right)} \partial^{k} \Phi(x)\right\|_{p} d t_{1} \ldots d t_{k} \leqslant c t^{k} m^{k}\|\Phi\|_{p} .
$$

С использованием леммы 3.5 доказательство обратной части теоремы 1 получается дословньм повторением рассуждения из $§ 4$ в [3] и поэтому здесь не приводится.

\section{Список литературы}

1. Никольский С. М., Лизоркин П. И. Приближение сферическими полиномами // Труды МИАН. 1984. Т. 166. С. $186-200$.

2. Никольский С. М., Лизоркин П. И. Функциональные пространства на сфоре, связанные с теорией приближений // Матем. заметки. 1987. Т. 41. № 4. С. 509-516.

3. Никольский С. М., Лизоркин П.И. Аппроксимация функций на сфере // Изв. АН СССР. Сер. матем. 1987. Т. 51. №3. С. 635-651.

4. Федоров В. М. Приближение функций на сфере // Вестник МГУ. Сер. матем., мех. 1990. № 1. C. $15-23$.

5. Рустамов Х. П. О приближении функций на сфере // Докл. АН СССР. 1991. Т. 320. №6. C. 1319-1325.

6. Тихомиров В. M. Теория приближений. Гл. $4, \S 2$ // Итоги науки и техники. Соврем. пробл. матем. Фунд. направления. Т. 14, 1987.

7. Камзолов А.И. Об интерполяционной формуле Рисса и неравенстве Бернштейна для функций на однородных пространствах // Матем. заметки. 1974. Т. 15. №6. С. 967-978.

8. Бессе А. Многообразия с замкнутьми геодезическими. М.: Мир, 1981.

9. Хелгасон $C$. Группы и геометрический анализ. М.: Мир, 1987.

10. Хелгасон $C$. Дифференциальная геометрия и симметрические пространства. М.: Мир, 1964.

11. Вейль $A$. Интегрирование в топологических группах и его применения. М.: ИЛ, 1950.

5 Математический сборник, т. 188, №5 
12. Бейтмен Г., Эрдейи А. Высшие трансцендентные функции. Т. 2. М.: Наука, 1974.

13. Гото М., Гроссханс Ф. Полупростые алгебры Ли. М.: Мир, 1981.

14. Бурбаки Н. Группы и алгебры Ли. Гл. VII-VIII. М.: Мир, 1978.

15. Воль Д Джс. Пространства постоянной кривизны. М.: Наука, 1982.

16. Никольский C. М. Приближение функций многих переменных и теоремы вложения. М.: Наука, 1977.

Петрозаводск

Поступила в редакцию

26.11 .1993 